\title{
Cloud Learning Platform Design and Implementation Under Wed2.0 Environment
}

\author{
Changcheng $\mathrm{Wu}$, Hongte Chen \\ College of Fundamental education, Sichuan Normal University, Chengdu 610066, China \\ awucc@163.com
}

Keywords: Cloud Learning, Cloud, Web2.0, Network Learning Community.

\begin{abstract}
Taking education as an example of discovery learning community to explore web2.0 environment design and build cloud learning platform. Based on the principles of SLL design, display SLL cloud learning platform system architecture, technology adoption Wordpress2.7 for supporting, relying on the "mix" mode to build, integrate Web2.0 service experience. Applications IRC, Google forums, Wiki, and promote a seamless link among the various SNS, pooling of resources also become easier.
\end{abstract}

\section{Background}

Under the continuous driving by network, a variety of online learning and teaching platform springing up, such as Moddle, CMS, Wordpress, Discuz, WebCL, WebCT, BlackBoard, DNN (DotNetNuke), are along with computer technology and network technology development designed to meet the educational needs of different network platforms. However, in the context of the development of web2.0 social software, online cloud learning platform gradually become civilians, popular, user-centered, organizational learning activities gradually communities and groups collective intelligence, collaborative creation, interaction, It has become the main theme of Teaching. Web2.0 environment online education, Flickr, Youtube, Google, Twitter, Wiki, Blog as the basis for the Panel, contacts, TAG, SNS, RSS as the elements used in network integration of education as the core, based on six times new forms of online education separate theory and technology. [1]

\section{SLL Introduction}

"SocialLearnLab" abbreviation as "SLL", Learning Community is a network collaborative learning community, led by the Beijing Normal University teacher XiuliZhuang, as freely organize partnership sponsors, projects and activities to practice the basic unit of community to introduce more people to the network and cloud learning, at the same time also calling community friends to work together to create an educational era of discovery. Community aim is to practice, study, dissemination socialization, connected learning. Socialization is found to connect learning to play individual creativity center to connect and dialogue as the basic approach to knowledge management as the basic approach to community representation. Through self-organized, helping community members to use a variety of ways online and offline, connected by increasing the individual, to promote community dialogue, through mining and play individual wisdom to promote the evolution of community-based organizations and decision-making, the realization of the community to promote the development of individual maximization to promote personal connection to optimize the development of the community. And dissemination of learning socialization connected ideas, methods, resources, tools, and the associated spread of free software education ideas, methods, resources and tools. SLL personnel involved in primary and secondary school teachers, teacher colleges, undergraduate, graduate and doctoral students, as well as friends interested in online education. [2] 


\section{SLL Cloud Learning Platform System Structure and Function}

SLL cloud learning platform around teams, projects, activities, themes, community members and to conduct collaborative authoring system design structure, members of the community to promote collaborative creation, knowledge sharing, cloud learn and grow together. SLL cloud learning platform system structure and function mainly consists of three parts, SLL theme module, SLL management module, SLL project cloud module. SLL learning theme module overview of the community and community projects, and to enter the site's users to navigate, user-friendly SLL learn about the community and the site itself. SLL Management module enables site management background, such as web-based management, user management, link management, appraisal management, labeling management, etc., improving the efficiency and functionality of the site. SLL project Cloud module uses a variety of social software and tools for composite applications, a member of the project to achieve cloud development. They include: collaborative discussion, co-authoring, real-time conferencing capabilities, provide a platform and technical support for the launch and implementation of the project.

\section{Achieve SLL Cloud Learning Platform}

We use Wordpress2.7 supported, Wordpress personalized service, be able to fight the good website publishing platform. In accordance with Wordpress website said: "WordPress is free open-source software, it can build a strong power network publishing platform, but more is applied to personalized blog for the blog application, which allows you not to worry about background technology, to concentrate on the content of the site. "technical barriers" [3] application Wordpress low and convenience, combined with GoogleCode, IRC, GoogleGroup, good-looking book, etc., relying on the" mix and match "mode of Web2.0 learning platform cloud network . So that there is a link between the various web2.0 site, but also more convenient pooling of resources. Relying SLL system architecture and modules, it further refined, to build a "educational discovery" cloud learning platform.

SLL Topic Module.SLL topic module is built by below three partial modules, Site Map, Community Dynamics and Project Summary.

Site Map. Firstly, SLL community presented in English name, Message, search boxes, etc. Secondly, the use of buttons to achieve the site navigation, content include: Home, About Us, teams, projects, experts, information, forums, English, WIKI, etc.

Community Dynamics.Through writing Blog and publishing web pages, the way to achieve a variety of display and update SLL community activities, work e-mail newsletters, community developments, news summaries, which is equivalent to Community Team newsletters and promotional Community Team role.

Project Summary. By Wordpress background operation, to achieve a summary of experts, books creation, translation projects, scientific research projects and more. After easy to open a Web page, a quick glance the main window project development and community dynamics, but also the community's external development community.

\section{SLL Management Module}

Logs and Web Management. Use Wordpress built SLL portals, mainly in the way of logging and web publishing information on the log, and Web pages create, modify, save, publish, etc., through the backlog and web management implementation.Background intuitive is interface, convenient, user-friendly platform maintenance and management.

Link Management.Management of various links, such as links to various social learning website, expert advisor blog, community promoters blog, friendship community while managing the link order, a link classification, open way, etc.

Subject Management.Wordpress interface presented in a different theme show, a theme present a different style. By changing themes and templates, to achieve style change, alter the overall 
interface layout, images, colors, fonts, title display position, columns and the like. By Admin Templates, Theme Options, Theme Editor, display style, column view, Widget and other modifications website front interface.

Tag and Category Management.Label and classification is one of the characteristics of web2.0, also has applications in Wordpress. SLL community in the form of articles published are based blog published articles for each article are based on the classification and labeling management, while automatic collection and classification list tag cloud is formed, easy to read and the author and the reader to find, but also allows the administrator management and maintenance.

Comments Management. Since the contents of the page in the form of logs released, so you want to comment to add, delete, save, etc. to manage. Combine to Mailbox to achieve the site easily and quickly manage.

User Management. To participate SLL team members in the registration, login and delete, user rights management, including five user permissions: Administrator, edit, author, contributor, subscriber. Administrators with the highest community management authority, to achieve the overall management of the site; Editing to achieve the website pages, blog management; to achieve release of the blog's author, writing and management of comments; subscribers can view the website and subscribe to the log.

Plug-in Management. After the Wordpress installation, it has good scalability endless supply of plug-in support. A variety of plug-in can help manage, maintain and optimize the site and achieve plug-in installation, upgrade, unloading etc.

Automatic Collection of Blog Management."BDP RSS Aggregator is the aggregation of Wordpress plug-in rss, directly in Sociallearnlab platform automatic collection of blog function." [4] blog pooled resources to achieve the collection team, but also facilitate the establishment of contacts and influence among users.

\section{SLL Project Cloud Module}

Based GoogleCode + SVN achieve WIKI creative people involved, and different versions of the control and project management. Use Everydo achieve the management of the project, GoogleGroup forum is also a mailing list, to achieve real-time discussions with members of non-Sociallearnlab exchange; IRC service implementation binding QQ, MSN, Gtalk, etc., and by creating Sociallearnlab the SLL channels to achieve Sociallearnlab community members in online discussions and communication, detail as below Fig:

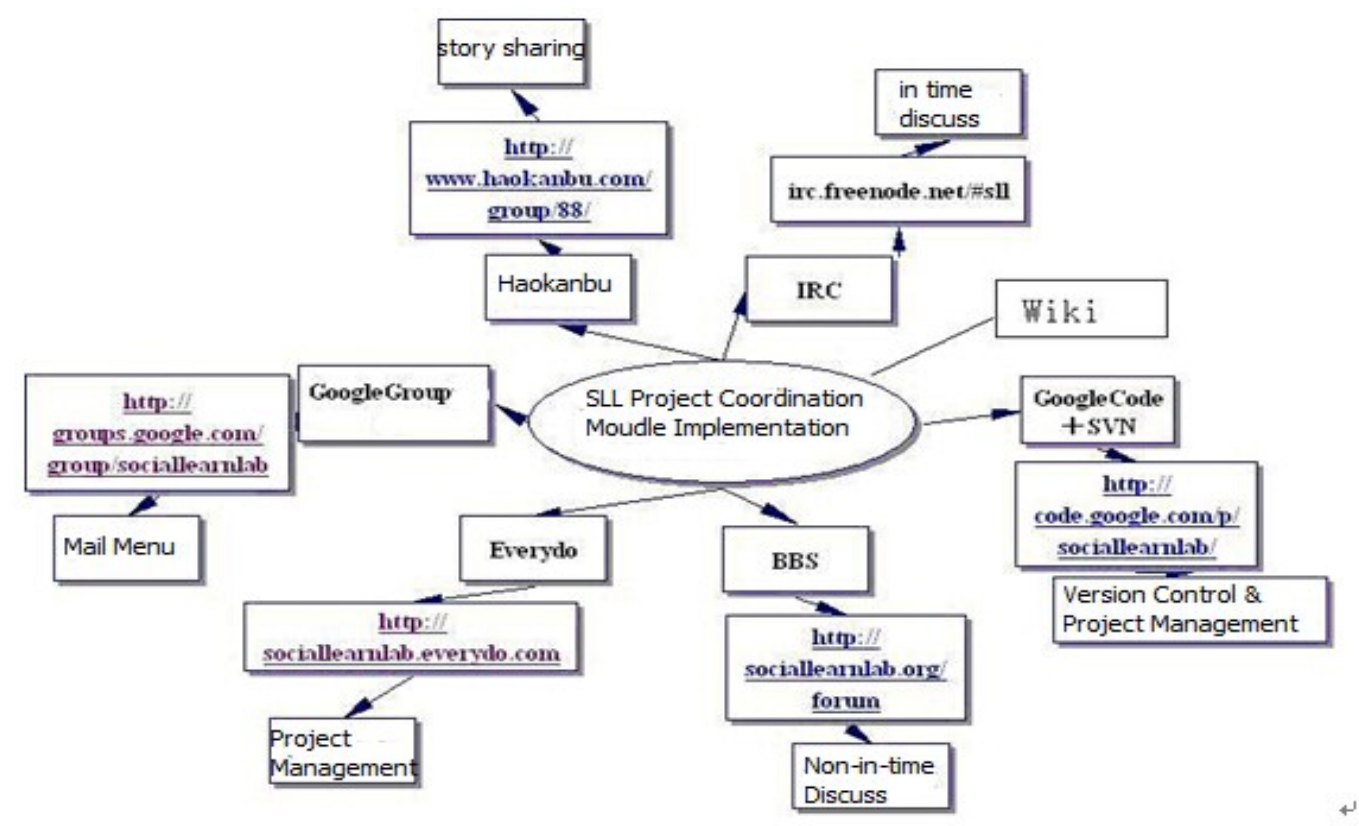

Fig1. SLL project cloud module Figure 
GoogleGroup Forum.Background operation by Wordpress, paste the following code into the Widgets sidebar can achieve Google Forum SocialLearnLab page shows, subscriptions, and link the code below:

<table border=0 style="background-color: \#fff; padding: 1px;" cellspacing=0>

$<$ tr $><$ td $><$ imgsrc="http://groups.google.com/groups/img/3nb/groups_bar.gif"

height=26 width=132 alt="Google Groups" $>$

$</ \mathrm{td}></ \operatorname{tr}><\operatorname{tr}><$ td style="padding-left: $1 \mathrm{px} ">$

Subscribe to "SocialLearnLab"

$</ \mathrm{td}></$ tr $><$ form

action="http://groups.google.com/group/sociallearnlab/boxsubscribe" $><\operatorname{tr}><\operatorname{td}$ style="padding-left: $1 \mathrm{px} ; ">$

Email: $<$ input type=text name=email $>$

$<$ input type=submit name="sub" value="submit" $></ \operatorname{td}></ \operatorname{tr}>$

$</$ form $><$ tr $><$ td

align=right $><$ a

href="http://groups.google.com/group/sociallearnlab">Visit

this group $</ \mathrm{a}></ \mathrm{td}></$ tr $></$ table $>$

Also, by adding the following code to add the Google forum to theme editor head.php files, to achieve the Forum in the navigation bar displaying:

$<\mathrm{li}><$ a href="https://groups.google.com/group/sociallearnlab"target=_blank $>$ GoogleGroup $</ \mathrm{a}>$

Everydo.Everydo community project created a total of SLL (http: //sociallearnlab/everydo.com/), the total project is again composed of various sub-projects, such as "social Application Notes (Students Version)" project and Google service applications "Handbook ", can be seen as Sociallearnlab subprojects, each subproject in Everydo able to view and make appropriate settings, you can create individual subprojects. These include: project updates, to-do tasks, calendar, project schedule, work logs, project view, project settings, project staff, task list (the task list), milestones, WordPad and so on. Implement the macro-management of the project.[5]

IRC.Under MS Windows and Linux environment, community members to achieve real-time conversation, we use IRC as a tool. IRC server by agreement and (Server) irc.freenode.net, fill in the user name and password, add the session, the user can enter the channel for real-time discussions and exchanges \#SLL.[6]

GoogleCode+SVN. Googlecode and SVN combination to achieve collective creation file version control, SLL's Google Code Address: http: //code.google.com/p/sociallearnlab/. To realize project membersmanagement, Wiki version control, Wiki collective creation. The main achievement of the SVN client version control, in the "copy, modify, merge," the principle drivers, the realization of the client install TortoiseSVN,the command: SVN server for local SVN consistent with Commit, Checkout, Del, Add like. In the Source directory Sociallearnlab SVN location is Googlecode core, SVN include the following directories: tasks unrelated to collect a variety of auxiliary and main project, supported by the nature of task code; otangle corresponding to the various members of the catalog open, collect the usual accumulation, after mature may be incorporated into the trunk; trunk main branch all the latest results of the organization in the interim, that is, our books; branches release version branch collect, collect initial results, and maintained for the release of the chase;wiki books.

Haokanbu.Haokanbu (www.haokanbu.com) uses graphic to achieve sharingteaching activities and participation of community members SLL story, so that the content of the original text is more vivid. Teachers and students by writing and participation, stories, tutorials, join studying group, share their learning, bringing together the collective wisdom.

WIKI Platform.SLL Learning Community Wiki platform built using Mediawiki, Mediawiki is free software written in PHP. Features of MediaWikiis perfection, learn sufficient information to establish the site of choice wiki program." [8] The use of Wikipedia platform collaborative creation projects, such as the completed manuscript, revise, and then improve, discussion, restore historical 
versions and so on. It can be used as resource gathering place, easy for users to find and use. Mainly it consists of four parts: The article, discusses, the source and history.

\section{Summary}

SLL learning platform for building community Web2.0 environment full advantage of a variety of social software is based on the use of splicing and integrated manner, as set up the "building blocks" of various Web2.0 services into SLL community platform. Community user-centric, user experience Web2.0 practice resources, the platform to provide a more complete functionality, external portals, development projects, the pooling of resources, building up a network, a comprehensive user web2.0 build a SSL Learning bridge between community practice and education.

\section{Acknowledgements}

General project from Sichuan Province Education Department: " The influence of self-organizing characteristics from network learning community on knowledge construction" (project number: 14SB0037); Scientific research project from Sichuan Normal University: "Network learning community practice research under the cloud environment" (project number: 12410002); Experimental technology and management project from Sichuan Normal University "The construction and application research under the cloud technology platform in elementary school science education" (project number: SYJS2013-07).

\section{Reference}

[1]Yongping Wong, Education Informatization Technology under Web2.0, [DB/OL]http://www.edu.cn/survey_1666/20070516/t20070516_232708.shtml

[2]About sociallearnlab, [DB/OL]http://sociallearnlab.org/?page_id=3

[3] wordpress, wordpress Brief introduction, [DB/OL]http://cn.wordpress.org/

[4]Boyu, WP RSS Aggregation widget-BDP RSS Aggregator,http://www.alexrun.com/

[5] Sociallearnlab,UsageEverydo, http://code.google.com/p/sociallearnlab/wiki/UsageEverydo

[6]WanqingWang, UsageIRC, http://code.google.com/p/sociallearnlab/wiki/UsageIRC

[7] GuangtaoXu, Inter-cloud learning platform design based on network, [DB/OL].61.144.60.222/0518/zuopin/kechenglunwen/8.doc

[8]WorldEncyclopedia,Whatis mediawiki? [DB/OL] http://www.allwiki.com/wiki/Mediawiki 